\title{
A Retrospective Study of the Correlation Between Hand Grip Strength and Functional Outcomes for Clients with Shoulder Pain
}

\author{
Andrew Ryan Lum ${ }^{1 *}$ and Carrie Ciro ${ }^{2}$ \\ ${ }^{1}$ OTD, MOT, OTR/L, Rockhurst university, Occupational Therapy Department, USA \\ ${ }^{2} \mathrm{PhD}$, OTR/L, FAOTA, University of Oklahoma, Department of Rehabilitation Services, USA
}

*Corresponding author: Andrew Ryan Lum, OTD, MOT, OTR/L, Rockhurst University, Occupational Therapy Department, USA

Received Date: January 06, 2020

Published Date: January 13, 2020

\footnotetext{
Abstract relationship is unclear.

Abbreviations:

- $\quad$ OT- Occupational therapist.

- $\quad$ EMG - Electromyography; UE - upper extremity.

- MCID- Minimally important clinical difference.

- $\quad$ SPADI- Shoulder Pain and Disability Index

- Lbs- Pounds.

- $\quad$ Kg-Kilograms.
}

Background: People with orthopedic shoulder injuries often have reductions in grip strength and function, but the correlation of these

Purpose: Explore the relationship between grip strength scores and functional outcome measures scores for patients experiencing shoulder pain and examine whether occupational therapy intervention was associated with positive changes in grip strength and self-reported function.

Methods: A retrospective cohort study examining the charts of patients seen in an outpatient clinic June 2014-July 2018, with the following inclusion criteria: people 18-85 years of age with acute/chronic shoulder pain, and/or rotator cuff pathology. Patients received therapeutic exercise/ activity; modalities; and functional training. Outcome measurements included baseline/discharge scores for grip strength and the Shoulder Pain and Disability Index (SPADI), a self-report of pain and disability with a total percentage score ranging from 0 to 100 , where $0=$ best and $100=$ worst.

Results: The records of 31 people met inclusion criteria. Grip strength increased ( $\left.x^{-}=7.6 \pm 11.1 \mathrm{lbs}\right)$; SPADI scores decreased (improved) by $26.6 \pm 19.7$ points. A moderate, negative correlation $(r=-0.596, P=.01)$ was found between initial affected grip strength scores and initial SPADI scores. Conversely, there was positive correlation $(\mathrm{r}=0.80, \mathrm{P}=.67)$ between post-intervention grip strength scores and SPADI scores.

Conclusion: Grip strength and SPADI scores improved after a course of occupational therapy. There was also a moderate correlation between pre-intervention grip strength and SPADI scores and a positive correlation between the post-intervention grip and SPADI scores.

Keywords: Grip strength; Occupational therapy; Upper extremity rehabilitation; SPADI

\section{Introduction}

The upper extremity is comprised of the shoulder complex, arm, forearm, and hand [1,2]. For the upper extremity to function in all ranges and across all tasks, muscle and joint structures need to work well collectively. Deficits in one muscle or joint structure may therefore lead to changes in movement, strength or function in other joints or in overall functional status [3].

Upper extremity performance and functional mobility is better, when the shoulder is stable and pain free [4]. Shoulder 
pain is the third most common musculoskeletal condition with incidence ranges up to $2.5 \%$ [5]. These proximal aspects of the upper extremity can affect functional performance of the hand [6]. Hand grip strength has been found to correlate with strength of other muscle groups and thus can be utilized as a good predictor of overall upper body strength as well as identify people at higher risk of physical disability [7-11]. Grip strength tends to increase into the fourth decade of life while declining at an accelerated rate thereafter [12]. The right hand is characteristically stronger than the left and men typically have higher grip strength than women in the same age range [13].

Changes in shoulder position, health, and integrity have adverse effects on grip strength $[2,4,6,7,10,14]$. During grip strength testing, the muscles of the rotator cuff help stabilize the humerus 4 as well as maintain scapular position in an ergonomically protracted state [6]. While the scapula is protracted, increased activation of muscles surrounding the scapula such as the serratus anterior, upper trapezius, as well as the flexor carpi ulnaris, flexor carpi radialis, and palmaris longus is observed and can further influence and improve grip strength measures [6]. When there is dysfunction in these muscles, through observation of decreased scapulohumeral rhythm and in combination with poor scapular position throughout movement, grip strength can reduce $13.14 \%$ in comparison to its norm for the individual [6]. Subsequently, fatigue in the upper extremity following a shoulder exhausting regime and recovery time, has led to a decrease in grip strength and was confirmed through electromyography (EMG) results as well as a reorganization of movement strategies [15]. This evidence illustrates a positive relation between shoulder function and grip strength force [10]. Application and utilization of this relationship could help with prediction of disability and functional limitations [9].

We sought to examine the correlational relationship between grip strength and self-reported functional outcomes in this study. In addition, we also sought to describe differences in strength and self-reported function after occupational therapy intervention in an outpatient setting. In our first aim, we examined the change in baseline and discharge scores between grip strength and selfreported function to assess for clinical and statistical significance. In the second aim, we examined correlations between baseline grip and self-reported function scores followed by discharge grip and self-reported function scores. We hypothesized that people with shoulder injury receiving a course of occupational therapy would not only demonstrate significant and meaningful change in these outcomes, but also that the outcomes would be moderately and significantly correlated.

\section{Methods}

A retrospective cohort design was used to examine a convenient sample of patient outcomes within an outpatient clinic in the University of Kansas Health System. Institutional Review Board reviews were conducted, and research was granted at both the University of Kansas Medical Center as well as the a Midwest hospital and The University of Kansas Medical Center to a Midwest university. Each recorded patient in this study had completed a full course of occupational therapy, including evaluation, treatment, and discharge within the dates of July 2014 to October 2018.

\section{Inclusion criteria}

1) males and females aged 18 to 85 , with one of the following shoulder diagnoses per physician report: rotator cuff pathology, acute shoulder pain, and chronic shoulder pain. If the shoulder pain/diagnosis was accompanied by comorbidities not limited to the shoulder, or was secondary to another reason for treatment, the subject would be excluded. Additional exclusion criteria included: a history of neck/back pathology (including physician diagnosed and/or self-report), history of cognitive impairment, history of a neurological diagnosis, history of peripheral nerve injury, previous treatment for the affected shoulder on acute and/or acute rehab units, as well as individuals with balance impairments. Inclusion and exclusion were based upon the medical diagnosis provided by the physician referral, a chart review, as well as through the subjective interview information that was obtained from the patient during their treatment. A licensed occupational therapist (OT) with $>5$ years of experience examined and treated all the patients in this cohort. The OT completed all outcome measures at baseline and post-intervention prior to discharge.

\section{Hand strength}

Measurement of grip strength was completed using a Jamar dynamometer following standardized procedures 9 with the handle in the middle position across a three-trial test. The minimally important clinical difference (MCID) for grip strength from baseline to discharge is between $11.02 \mathrm{lbs}(5.0 \mathrm{~kg})$ and $14.5 \mathrm{lbs}(6.5 \mathrm{~kg})$ [16].

\section{Shoulder Pain and Disability Index (SPADI)}

The SPADI is a self-reported questionnaire developed to measure the pain and function associated with shoulder pathology for people with shoulder pain of musculoskeletal, neurogenic, or undetermined origin. It consists of 13 items in two domains: pain (pain symptoms, 5 items) and disability (physical function, 8 items). The items of both domains were scored on a visual analog scale ranging from 0 to 10 , where $0=$ no pain/no difficulty and 10 = worst pain imaginable/so difficult required help. Each domain score was equally weighted, then added for a total percentage score ranging from 0 to 100 , where $0=$ best (no pain and functioning normally) and $100=$ worst (extreme pain levels and complete functional impairment) [17]. The MCID for pre-intervention and post-intervention scores is 13 points [17].

\section{Therapeutic Procedure}

To better understand the outcomes, the procedures for assessment and intervention are outlined. An evaluation was completed in the first session of occupational therapy services.

The evaluation consisted of:

- Gathering of background information/occupational profile through a thorough chart review and subsequent interview. 
- Measurements of active range of motion with goniometer (shoulder flexion, shoulder abduction, internal rotation, external rotation, and shoulder extension).

- Gross manual muscle testing of the shoulder on a 0 to 5 grading scale (shoulder flexion, shoulder abduction, internal, and external rotation).

- Manual orthopedic testing (e.g., Neer's Impingement Test, Empty Can Test, Subscapularis Liftoff Test, Drop Arm Test, and Neural Tension Test) [18].

- $\quad$ Grip strength assessment.

- A self-reported rating of function using the SPADI.

Individualized goals were established based on the patient deficits, strengths and goals. Therapeutic interventions aligned with individual injury status and goals. Interventions consisted of therapeutic exercise; therapeutic activity (e.g., the Kinesio Tape Method, patient education); physical agent modalities (e.g., ultrasound, cold packs); Activity of Daily Living retraining (e.g., sleeping posture, bed mobility, overhead cleaning tasks); Instrumental Activity of Daily Living training; and work simulation. No specific grip exercises were provided or recommended.

\section{Data Analysis}

Due to the analysis of multiple factors and aims for this study, several statistical tests were run. Descriptive statistics were completed through a comparison of means within the sample $(n=31)$. These statistics were utilized to determine means for age, diagnosis, and visits. Paired sample t-tests were utilized between baseline and post-intervention SPADI scores as well as between baseline and post-intervention affected UE grip strength measures $(\mathrm{p}<0.05)$. Spearman's Rho's correlation was utilized for examining the relationships between strength and function through baseline affected UE grip strength measures and SPADI scores as well as between the post-intervention affected UE grip strength measures and SPADI scores $(\mathrm{p}<0.05)$.

\section{Result}

An extensive chart review was conducted on complete patient charts dated from June 2014 to October 2018. Thirty-one patients met study criteria varying in range from 24 to 85 years with an average age of $59.1 \pm 13.2$ years; $39 \%$ (12/31) were male and $61 \%(19 / 31)$ were female. Patients were seen for a minimum of 3 visits and a maximum of 30 visits but averaged $8.32 \pm 6.4$ visits per treatment course (Table 1). By diagnosis, 55\% (17/31) of patients presented with complaints of acute shoulder pain, $22.5 \%$ (7/31) chronic shoulder pain and $22.5 \%$ (7/31) with rotator cuff pathology, as diagnosed by the referring physician.

Across males and females, the baseline mean grip strength scores were $\left(\mathrm{x}^{-}=74.9\right.$, SD: 32.1 lbs.; Range 10.3-151.7lbs. Mean discharge grip strength scores were $\left(x^{-}=82.5\right.$, SD: 31.2lbs; Range 33.4-155.3lbs). Pre- and post-intervention grip scores were significantly improved $(\mathrm{p}=0.001)$. Because male and female scores are typically different, we also analyzed grip strength changes between males and females. Mean male baseline grip scores ( $\mathrm{x}$ =104.8; SD: 22.8lbs) were statistically different $(\mathrm{p}=0.019)$ from mean discharge grip scores ( $\mathrm{x}^{-}=114.3$; SD: 21.4lbs). Mean female baseline grip scores $\left(\mathrm{x}^{-}=55.9 ; \mathrm{SD}\right.$ : 21.1lbs) were statistically different $(\mathrm{p}=0.029)$ from mean discharge grip scores $\left(\mathrm{x}^{-}=62.1\right.$; $\mathrm{SD}$ : 15.2lbs).

In our second outcome, the mean baseline SPADI scores were ( $\mathrm{x}^{-}=37.7$, SD: 21.6; Range 11-86). Following the completion of treatment, Table 1 highlights the mean discharge SPADI scores were $(x=11, S D: 11.4$; range $0-53)$ which was significant $(p=0.00)$.

Table 1: Sample Characteristics $(\mathrm{N}=31)$ and Outcome Measurements at Baseline and Discharge.

\begin{tabular}{|c|c|c|c|c|}
\hline & Baseline Mean (SD) & Discharge Mean (SD) & Change Score Mean (SD) & p Value \\
\hline Age (years) & $59.06 \pm 13.2$ & NA & NA & NA \\
\hline Number of Visits & NA & $8.32 \pm 6.4$ & $-7.6 \pm 11.1$ & 0.001 \\
\hline $\begin{array}{c}\text { Baseline SPADI Scores - Discharge SPADI } \\
\text { Scores }\end{array}$ & $37.7 \pm 21.6$ & $11.0 \pm 11.4$ & $26.7 \pm 19.7 \mathrm{lbs}$ & 0.001 \\
\hline $\begin{array}{c}\text { Affected UE Baseline Grip Strength - Affected } \\
\text { UE Discharge Grip Strength }\end{array}$ & $74.9 \pm 32.1$ & $82.5 \pm 31.2 \mathrm{lbs}$ & & NA \\
\hline
\end{tabular}

$\mathrm{SD}=$ standard deviation; UE= upper extremity; SPADI=Shoulder Pain and Disability Index; NA=Not applicable.

Table 2: Non-Parametric Correlation of Affected UE Grip Strength with SPADI Scores (Spearman Rho's Correlation).

\begin{tabular}{|c|c|c|c|}
\hline \multirow{2}{*}{ Initial SPADI Score } & & Initial SPADI Score & Affected UE Initial Grip Strength \\
\hline \multirow{2}{*}{ Affected UE Initial Grips Strength } & Correlation Coefficient & 1 & $-.596^{* *}$ \\
\cline { 2 - 4 } & Sig. (2-tailed) & - & 0 \\
\hline \multirow{2}{*}{ Discharge SPADI Score } & Correlation Coefficient & $-.596^{* *}$ & 1 \\
\cline { 2 - 4 } & Sig. (2-tailed) & 0 & \\
\cline { 2 - 4 } Affected UE Discharge Grip Strength & Correlation Coefficient & 1 & 0.8 \\
\cline { 2 - 4 } & Sig. (2-tailed) & 0.8 & 0.669 \\
\cline { 2 - 4 } & Correlation Coefficient & & 1 \\
\hline
\end{tabular}

**. Correlation is significant at the 0.05 level (2-tailed). 
A Spearman's Rho correlation test was run to observe the relationship between the initial SPADI scores and the initial affected UE grip strength scores. A moderate, negative correlation of $r=-0.596(p=0.00)$ was found between the two scores (Table 2). A second Spearman's Rho correlation was completed with the discharge scores of both the affected UE grip strength and SPADI scores respectively. This produced a non-significant $(p=0.67)$, correlation of $r=0.80$ between the two measures.

\section{Discussion}

With this study, we wanted to describe differences in grip strength and self-reported function after a typical occupational therapy intervention in an outpatient setting. We found that grip strength and self-reported function clinically and statistically improved from baseline to discharge. In our second aim, we sought to examine the correlational relationship between grip strength and self-reported functional outcomes at baseline and discharge. Our findings support a moderate, inverse relationship where the lower the grip score, the higher (worse) the self-reported function score. At discharge, we found a positive relationship between discharge grip and self-reported function although the outcomes were not significant.

As noted above, grip strength of the affected UE experienced a positive and significant change. Other studies have found similar results with grip strength of the affected UE following therapeutic intervention. Stock, et al. [19] found that post stroke, grip strength will improve with significance within the first year following therapeutic intervention. This study did focus on grip strength for some of its interventions though, while our study did not. Likewise, a study completed by Bridges, et al. [20] showed a $465 \%$ improvement in grip strength post intervention for patients that presented with cervical myelopathy. This study similarly focused on conservative management treatments; but was provided in the physical therapy practice versus occupational therapy. The different practice and knowledgebase may have provided the subjects different intervention techniques than those completed in our study.

SPADI scores experienced the greatest change which resulted in double the score needed for a clinically significant change.15 Not surprisingly, other studies using the SPADI have found similar results. Crowell, et al. [21] also observed a significant improvement in SPADI scores following manual orthopedic physical therapy intervention for patients with glenohumeral osteoarthritis. Similarly, a study completed by Ergönenç, et al. [22] examined the effects of ultrasound-guided suprascapular nerve pulsed radiofrequency on chronic shoulder pain which revealed a significant improvement in SPADI scores following the intervention. Our work demonstrates the potential for occupational therapy to improve both grip strength and self-reported function in people with acute and chronic orthopedic shoulder diagnoses.

As predicted, we found a significant, moderate, and negative correlation between baseline grip and SPADI scores. This correlation indicated that there was a significant relationship between a lower grip strength score and a higher (poorer) SPADI score. Boissy, et al. [23] found a similar relationship in chronic stroke patients in that grip strength was an effective outcome measure tool in assessing upper extremity function. This study focused on more general functionally outcome measures though such as: the Fugl-Meyer, The Upper Extremity Performance Test for the Elderly (TEMPA), the Box and Block test, as well as the Finger-to-Nose test. Our test focused solely on the SPADI as the outcome measure but did produce similar results to functional improvement.

Correlations between discharge grip strength and SPADI scores yielded a stronger relationship in comparison to the pre-intervention data; but was not statistically significant. Intuitively, it follows that stronger grip scores may correlate strongly with improved function scores. These results did align with several studies such as that completed by Nascimento, et al. [24], which demonstrated how strengthening of the scapular stabilizing muscles in patients that have been experiencing chronic hemiparesis status post stroke leads to improved grip strength. Another study that found several correlations of grip strength and the functional outcomes of patients was completed by Schwartz, et al. [25]. Her study found multiple correlations between grip strength improvements and patients' self-reported, subjective evaluations.

\section{Conclusion}

Occupational therapy is designed to help promote and improve functional independence. Unsurprisingly, the results showed that a course of occupational therapy for people with orthopedic shoulder injuries may result in greater functional independence as well as grip strength. The results also yielded a moderate relationship between initial grip strength scores and initial SPADI scores, as well as a positive non-significant relationship between the post-intervention grip strength and SPADI. Due to limitations in the small and convenient sample, researchers should continue to explore further distinctions in the relationship between shoulder health, grip strength, and functional performance.

\section{Acknowledgment}

None.

\section{Conflict of Interest}

No conflict of interest.

\section{References}

1. Yoo WG (2013) Changes in acromion and scapular position after shortterm overhead work. J Phys Ther Sci 25(6): 679-680.

2. Su CY, Lin JH, Chien TH, Cheng KF, Sung YT (1994) Grip strength in different positions of elbow and shoulder. Arch Phys Med Rehabil 75(7): 812-815.

3. Norman K, Stobäus N, Gonzalez MC, Schulzke JD, Pirlich M (2011) Hand grip strength: outcome predictor and marker of nutritional status. Clin Nutr 30(2): 135-142.

4. Shim J, Park M, Lee S, Lee M, Kim H (2010) The effects of shoulder stabilization exercise and shoulder isometric resistance exercise on shoulder stability and hand function. Journal of Physical Therapy Science 22(3): 227-232. 
5. Lazarus M, Rynning R (2011) Anatomy and kinesiology of the shoulder. In TM Skriven, AL Osterman, JM Fedorczyk, PC Amadio (eds.), Rehabilitation of the hand and upper extremity ( $6^{\text {th }}$ edn) PA: Elsevier Mosby, USA, pp. 66-67.

6. Yang J, Lee J, Lee B, Jeon S, Han B, Han D (2014) The effects of active scapular protraction on the muscle activation and function of the upper extremity. J Phys Ther Sci 26(4): 599-603.

7. Mercierand C, Bourbonnais D (2004) Relative shoulder flexor and handgrip strength is related to upper limb function after stroke. Clin Rehabil 18(2): 215-221.

8. Rantanen T, Era P, Kauppinen M, Heikkinen E (1994) Maximal isometric muscle strength and socioeconomic status, health, and physical activity in 75-year-old persons. Age Ageing 2(3): 206-220.

9. Rantanen T, Guralnik JM, Foley D, Masaki K, Leveille S, Curb JD, White L (1999) Midlife hand grip strength as a predictor of old age disability. JAMA 281(6): 558-560.

10. Trambadia J, Khushbu B, Praful B, Vishal K (2013) A Study to Find Correlation of Grip Strength and Coordination on Upper Extremity Functions in Stroke Patients. Indian Journal of Physiotherapy and Occupational Therapy 7(2): 186-190.

11. Wind AE, Takken T, Helders PJ, Engelbert RH (2010) Is grip strength a predictor for total muscle strength in healthy children, adolescents, and young adults? Eur J Pediatr 169(3): 281-287.

12. Kallman DA, Plato CC, Tobin JD (1990) The role of muscle loss in the age-related decline of grip strength: cross-sectional and longitudinal perspectives. J Gerontol 45(3): M82-M88.

13. Mathiowetz V, Kashman N, Volland G, Weber K, Dowe M, Rogers S (1985) Grip and pinch strength: normative data for adults. Arch Phys Med Rehabil 66(2): 69-74.

14. Kinali G, Kara S, Yıldırım MS (2016) Electromyographic analysis of an ergonomic risk factor: overhead work. Journal of physical therapy science 28(6): 1924-1927.
15. Hawkes D, Grant M, Mc Mahon J, Horsley I, Khaiyat O (2018) Can grip strength be used as a surrogate marker to monitor recovery from shoulder fatigue? J Electromyogr Kinesiol 41: 139-146.

16. Bohannon RW (2019) Minimal clinically important difference for grip strength: a systematic review. J Phys Ther Sci 31(1): 75-78.

17. Roy JS, Mac Dermid JC, Woodhouse LJ (2009) Measuring shoulder function: a systematic review of four questionnaires. Arthritis Rheum 61(5): 623-632.

18. Konin JG, Lebsack D, Valier AS, Isear JA (2006) Special tests for orthopedic examination. NJ: SLACK Incorporated, USA, pp. 24-85.

19. Stock R, Thrane G, Askim T, Anke A, Mork PJ (2019) Development of grip strength during the first year after stroke. J Rehabil Med 51(4): 248-256.

20. Bridges J, Sandoval R (2018) Clinical outcomes following conservative management of chronic traumatic cervical myelopathy: A case report. Physiother Theory Pract 34(3): 231-240.

21. Crowell MS, Tragord BS (2015) Orthopaedic manual physical therapy for shoulder pain and impaired movement in a patient with glenohumeral joint osteoarthritis: a case report. J Orthop Sports Phys Ther 45(6): 453461.

22. Ergönenç T, Beyaz SG (2018) Effects of ultrasound-guided suprascapular nerve pulsed radiofrequency on chronic shoulder pain. Med Ultrason 20(4): 461-466.

23. Boissy P, Bourbonnais D, Carlotti MM, Gravel D, Arsenault BA (1999) Maximal grip force in chronic stroke subjects and its relationship to global upper extremity function. Clin Rehabil 13(4): 354-362.

24. Nascimento LR, Polese JC, Faria CD, Teixeira Salmela LF (2012) Isometric hand grip strength correlated with isokinetic data of the shoulder stabilizers in individuals with chronic stroke. J Bodyw Mov Ther 16(3): 275-280.

25. Schwartz DA, Tyler H, Adams J, Ellis B (2005) What can handgrip strength tell the therapist about hand function? British Journal of Hand Therapy 18(4): 457. 